\title{
Controllability problem for fractional integrodifferential evolution systems of mixed type with the measure of noncompactness
}

\author{
Haiyong Qin, Jianwei Liu* and Xin Zuo
}

"Correspondence: liujw@cup.edu.cn Department of Automation, China University of Petroleum (Beijing), Beijing, 102249, People's Republic of China

\begin{abstract}
We consider the controllability problem for a class of fractional evolution systems of mixed type in an infinite dimensional Banach space. The existence of mild solutions and controllability results are discussed by a new estimation technique of the measure of noncompactness and a fixed point theorem with respect to a convex-power condensing operator. However, the main results do not need any restrictive conditions on estimated parameters of the measure of noncompactness. Since we do not assume that the semigroup is compact and other conditions are more general, the outcomes we obtain here improve and generalize many known controllability results. An example is also given to demonstrate the applications of our main results.
\end{abstract}

MSC: 26A33; 34B15; 93B05; 93C25

Keywords: controllability; fixed point theorem; Banach space; fractional evolution system

\section{Introduction}

The concept of controllability was firstly introduced by Kalman in 1960. There has been a significant development in controllability results of systems represented by differential equations, integrodifferential equations, impulsive equations, differential inclusions, neutral differential equations and delay differential equations in Banach spaces. Most of the previous results require the assumption that the operator semigroup $T(t)$ is compact. Using a compact analytic semigroup and a nonlinear alterative of Leray-Schauder type for multivalued maps due to O'Regan, Yan [1] established sufficient conditions for the controllability of fractional order partial neutral functional integrodifferential inclusion with infinite delay. Balachandran and Park [2] studied the controllability of fractional integrodifferential systems in Banach spaces, and an example with a compact analytic semigroup was also given. Based upon Bohnenblust-Karlin's fixed point theorem and a compact semigroup, Chang [3] investigated a controllability result of mixed Volterra-Fredholm type integrodifferential inclusions in Banach spaces. Chalishajar [4] considered sufficient conditions for semilinear mixed Volterra-Fredholm type integrodifferential systems in a Banach space via a compact semigroup. Hernández and O'Regan [5] pointed out that the control-

○2014 Qin et al.; licensee Springer. This is an Open Access article distributed under the terms of the Creative Commons Attribution License (http://creativecommons.org/licenses/by/2.0), which permits unrestricted use, distribution, and reproduction in any medium, provided the original work is properly cited. 
lability results will be restricted to the finite dimensional space when the compactness of a semigroup and some other assumptions are satisfied. So, many researchers have tried to get sufficient conditions guaranteeing the controllability results of various systems without involving the compactness of a semigroup.

Wang et al. [6] studied the following fractional neutral differential system in an abstract space:

$$
\left\{\begin{array}{l}
{ }^{C} D_{t}^{q}\left(x(t)+F\left(t, x_{t}\right)\right)+A x(t)=C u(t)+G\left(t, x_{t}\right), \quad t \in(0, a] \\
x_{0}(\theta)=\phi(\theta), \quad \theta \in(-\infty, 0] .
\end{array}\right.
$$

By using the fractional power of operators and Sadovskii's fixed point theorem, they obtained the complete controllability of fractional neutral differential systems in an abstract space without involving the compactness of characteristic solution operators, but the main results require that the set $\Pi_{h, \delta}(t)$ is relatively in a Banach space $\mathbb{X}$ for arbitrary $h \in(0, t)$ and $\delta>0$ (see $\left(H_{4}\right)$ in [6]).

Fečkan et al. [7] discussed the controllability of a class of fractional functional evolution equations of Sobolev type

$$
\left\{\begin{array}{l}
{ }_{0}^{C} D_{t}^{q}(E x(t))+A x(t)=f\left(t, x_{t}\right)+B u(t), \quad t \in J:=[0, a], \\
x(t)=\phi(t), \quad-r \leq t \leq 0
\end{array}\right.
$$

in a Banach space. With the help of two new characteristic solution operators and their properties, such as compactness and boundedness, the controllability results for fractional evolution equations were obtained by the Schauder fixed point theorem. Later, researchers have always tried to avoid the compactness of a semigroup via the measure of noncompactness. Ji et al. [8] considered the controllability of impulsive functional differential equations with nonlocal conditions by the measure of noncompactness and the Mönch fixed point theorem. Machado et al. [9] obtained the controllability results for a class of abstract impulsive mixed-type functional integrodifferential equations with finite delay in Banach spaces, sufficient conditions for controllability were obtained by the Mönch fixed point theorem via the measure of noncompactness and semigroup theory. Those results in $[8$, 9] do not assume the compactness of the evolution system, but restrictive conditions on the estimated parameter of the measure of noncompactness are required.

By using the techniques of convex-power condensing operators, Zhu et al. [10] considered the existence of mild solutions for one order impulsive semilinear differential equations with nonlocal conditions. Xue [11] obtained the existence results of integral solutions for nonlinear one order differential equations with nonlocal initial value conditions under the assumptions of the measure of noncompactness in a separable and uniformly smooth Banach space. Ahmad et al. [12] showed the existence of mild solutions to one order impulsive integrodifferential equations with the nonlinearity of a form $f(t, u(t), G u(t))$, where $G u(t)$ represents a Volterra-type integral operator. Very recently, Wang and Zhou [13] investigated the complete controllability of fractional semilinear systems in infinite dimensional spaces of the type

$$
\left\{\begin{array}{l}
{ }^{C} D_{t}^{q} x(t)=A x(t)+f\left(t, x_{t}\right)+B u(t), \quad t \in J:=[0, b] \\
x(t)=x_{0} \in \mathbb{X}
\end{array}\right.
$$


Chen and Li [14] studied a nonlocal problem for fractional evolution equations of mixed type in a Banach space, the existence of mild solutions and positive mild solutions was obtained by utilizing the measure of noncompactness and a new fixed point theorem.

Inspired by the above results, here we consider the controllability of the following fractional evolution system of mixed type in an infinite dimensional Banach space $\mathbb{X}$ :

$$
\left\{\begin{array}{l}
{ }^{C} D_{t}^{q} x(t)+A x(t)=f(t, x(t),(S x)(t),(T x)(t))+B u(t), \quad t \in I=[0, b] \\
x(0)+g(x)=x_{0},
\end{array}\right.
$$

where ${ }^{C} D_{t}^{q}$ is the Caputo fractional derivative. $0<q<1, A: D(A) \subset \mathbb{X} \rightarrow \mathbb{X}$ is a closed linear operator and $-A$ generates a uniformly bounded $C_{0}$-semigroup $(T(t))_{t \geq 0}$ in $\mathbb{X}$. $f$ and $g$ will be specified later. $B: U \rightarrow \mathbb{X}$ is a bounded linear operator, $u \in L^{2}[I, U]$, and integral operators $S$ and $T$ are defined as

$$
(S x)(t)=\int_{0}^{t} k(t, s) x(s) d s, \quad(T x)(t)=\int_{0}^{b} h(t, s) x(s) d s .
$$

Here $k \in C\left[\Delta_{1}, \mathbb{R}^{+}\right], \Delta_{1}=\{(t, s) \mid 0 \leq s \leq t \leq b\}$, and $h \in C\left(\Delta_{2}, \mathbb{R}^{+}\right), \Delta_{2}=\{(t, s) \mid 0 \leq t, s \leq$ b).

In the present paper, we introduce a suitable concept of mild solutions for abstract control system (1.1). Under some necessary conditions on the characteristic solution operators $\mathcal{S}_{q}(\cdot)$ and $\mathcal{T}_{q}(\cdot)$, we obtain the sufficient conditions of controllability results for system (1.1) when the operator $T(t), t>0$, is not compact. The methods we use are a new estimation technique of the measure of noncompactness and a fixed point theorem with respect to a convex-power condensing operator. The main results do not require any restrictive conditions on estimated parameters of the measure of noncompactness, i.e., parameters $L_{i}$ $(i=1,2,3)$ do not appear in inequality (3.1) and any other inequalities, which is the main difference between our study and the previous results, and also the main contribution of this paper.

The rest of the paper is organized as follows. In Section 2, we present some preliminaries and lemmas that are to be used later to prove our main results. In Section 3, we discuss the controllability results for system (1.1). At last, an example is provided to illustrate the theory in Section 4. Section 5 is a conclusion.

\section{Preliminaries and lemmas}

For the convenience of the readers, we shall recall here some necessary definitions from fractional calculus theory and some properties of the measure of noncompactness, one can refer to the monographs by Podlubny [15], Miller and Ross [16] and Deimling [17].

In this paper, we denote by $\mathbb{X}$ a Banach space with the norm $\|\cdot\|$. Assume that $\mathbb{Y}$ is another Banach space, $L_{b}(\mathbb{X}, \mathbb{Y})$ denotes the space of bounded linear operators from $\mathbb{X}$ to $\mathbb{Y}$. We also use $\|f\|_{L^{p}\left(I, \mathbb{R}^{+}\right)}$to denote the norm of $f$ whenever $f \in L^{p}\left(I, \mathbb{R}^{+}\right), 1 \leq p<\infty$. Let $L^{p}(I, \mathbb{X})$ denote the Banach space of functions $f: I \rightarrow \mathbb{X}$ which are Bochner integrable normed by $\|\cdot\|_{L^{p}(I, \mathbb{X})} \cdot C(I, \mathbb{X})$ represents a Banach space endowed with supnorm, i.e., $\|x\|_{C} \equiv \sup _{t \in I}\|x(t)\|$ for $x \in C(I, \mathbb{X})$. 
Definition 2.1 The fractional integral of order $q>0$ with the lower limit zero for a function $x \in L^{1}(I, \mathbb{X})$ is defined as

$$
I_{t}^{q} x(t)=\frac{1}{\Gamma(q)} \int_{0}^{t}(t-s)^{q-1} x(s) d s
$$

where $\Gamma(\cdot)$ is the Euler gamma function.

Definition 2.2 The Caputo fractional derivative of order $q>0$ with the lower limit zero for a functional $x$ is defined as

$$
{ }^{C} D_{t}^{q} x(t)=\frac{1}{\Gamma(n-q)} \int_{0}^{t}(t-s)^{n-q-1} x^{(n)}(s) d s, \quad 0 \leq n-1<q<n,
$$

where the function $x(t)$ has absolutely continuous derivatives up to order $n-1$.

Definition 2.3 (see [13]) A functional $x \in C(I, \mathbb{X})$ is said to be a mild solution of system (1.1) if it satisfies

$$
\begin{aligned}
x(t)= & \mathcal{T}_{q}(t)\left(x_{0}-g(x)\right) \\
& +\int_{0}^{t}(t-s)^{q-1} \mathcal{S}_{q}(t-s)[f(s, x(s),(S x)(s),(T x)(s))+B u(s)] d s,
\end{aligned}
$$

where operators $\mathcal{T}_{q}(t)$ and $\mathcal{S}_{q}(t)$ are defined by

$$
\mathcal{T}_{q}(t) x=\int_{0}^{\infty} h_{q}(s) T\left(t^{q} s\right) x d s, \quad \mathcal{S}_{q}(t) x=q \int_{0}^{\infty} s h_{q}(s) T\left(t^{q} s\right) x d s,
$$

where

$$
h_{q}(s)=\frac{1}{\pi q} \sum_{n=1}^{\infty}(-s)^{n-1} \frac{\Gamma(n q+1)}{n !} \sin (n \pi q), \quad s \in(0, \infty)
$$

is the functional of Wright type defined on $(0, \infty)$ which satisfies

$$
\begin{aligned}
& h_{q}(s) \geq 0, \quad s \in(0, \infty), \quad \int_{0}^{\infty} h_{q}(s) d s=1, \\
& \int_{0}^{\infty} s^{v} h_{q}(s) d s=\frac{\Gamma(1+v)}{\Gamma(1+q v)}, \quad v \in[0,1] .
\end{aligned}
$$

Lemma 2.1 (see [14]) The operators $\mathcal{T}_{q}(t), \mathcal{S}_{q}(t)(t \geq 0)$ have the following properties:

(1) For any fixed $t \geq 0, \mathcal{T}_{q}(t)$ and $\mathcal{S}_{q}(t)$ are linear bounded operators, i.e., for any $x \in \mathbb{X}$

$$
\left\|\mathcal{T}_{q}(t) x\right\| \leq M_{1}\|x\|, \quad\left\|\mathcal{S}_{q}(t) x\right\| \leq \frac{q M_{1}}{\Gamma(1+q)}\|x\|=\frac{M_{1}}{\Gamma(q)}\|x\|,
$$

where $M_{1}$ is a positive constant to be specified later. 
(2) The operators $\mathcal{T}_{q}(t)(t \geq 0)$ and $\mathcal{S}_{q}(t)(t \geq 0)$ are strongly continuous. Therefore, for all $x \in \mathbb{X}$ and $0 \leq t^{\prime} \leq t^{\prime \prime} \leq b$, one has

$$
\left\|\mathcal{T}_{q}\left(t^{\prime \prime}\right) x-\mathcal{T}_{q}\left(t^{\prime}\right) x\right\| \rightarrow 0, \quad\left\|\mathcal{S}_{q}\left(t^{\prime \prime}\right) x-\mathcal{S}_{q}\left(t^{\prime}\right) x\right\| \rightarrow 0 \quad \text { as } t^{\prime \prime} \rightarrow t^{\prime}
$$

Lemma 2.2 Let $\mathbb{X}$ be a Banach space and $\Omega_{\mathbb{X}}$ the bounded set of $\mathbb{X}$. The Kuratowski measure of noncompactness is the map $\partial: \Omega_{\mathbb{X}} \rightarrow[0,+\infty)$ defined by

$$
\partial(D)=\inf \left\{d>0: D \subseteq \bigcup_{i=1}^{n} D_{i} \text { and } \operatorname{diam}\left(D_{i}\right) \leq d\right\}, \quad \text { here } D \in \Omega_{\mathbb{X}} .
$$

Lemma 2.3 Let $D_{1}, D_{2}$ be two bounded sets of a Banach space $\mathbb{X}$. Then:

(1) $\partial\left(D_{1}\right)=0$ if and only if $D_{1}$ is relatively compact.

(2) $\partial\left(D_{1}\right) \leq \partial\left(D_{2}\right)$ if $D_{1} \subseteq D_{2}$.

(3) $\partial\left(D_{1}+D_{2}\right) \leq \partial\left(D_{1}\right)+\partial\left(D_{2}\right)$.

Lemma 2.4 (see [18]) Let $\mathbb{X}$ be a Banach space and $D \subset C(I, \mathbb{X})$ be bounded and equicontinuous. Then $\partial(D(t))$ is continuous on $I$, and

$$
\partial(D)=\max _{t \in I} \partial(D(t))=\partial(D(I))
$$

Lemma 2.5 (see [19]) Let $\mathbb{X}$ be a Banach space, let $D=\left\{x_{n}\right\} \subset C(I, \mathbb{X})$ be a bounded and countable set. Then $\partial(D(t))$ is Lebesgue integral on $I$, and

$$
\partial\left(\left\{\int_{I} x_{n}(t) d t \mid n \in \mathbb{N}\right\}\right) \leq 2 \int_{I} \partial(D(t)) d t
$$

Lemma 2.6 (see [14]) Let $\mathbb{X}$ be a Banach space, $D \subset \mathbb{X}$ be bounded. Then there exists a countable $D_{0} \subset D$ such that $\partial(D) \leq 2 \partial\left(D_{0}\right)$.

Lemma 2.7 (Fixed point theorem with respect to a convex-power condensing operator, see [20]) Let $\mathbb{X}$ be a Banach space, let $D \subset E$ be bounded, closed and convex. Suppose that $Q: D \rightarrow D$ is a continuous operator and $Q(D)$ is bounded. For any $S \subset D$ and $x_{0} \in D$, set

$$
\begin{aligned}
& Q^{\left(1, x_{0}\right)}(S) \equiv Q(S) \\
& Q^{\left(n, x_{0}\right)}(S) \equiv Q\left(\overline{c o}\left\{Q^{\left(n-1, x_{0}\right)}(S), x_{0}\right\}\right), \quad n=2,3, \ldots
\end{aligned}
$$

If there exist $x_{0} \in D$ and a positive integer $n_{0}$ such that for any bounded and noncompact subset $S \subset D$,

$$
\partial\left(Q^{\left(n_{0}, x_{0}\right)}(S)\right)<\partial(S)
$$

then $Q$ has at least one fixed point in D.

Definition 2.4 The fractional system (1.1) is said to be controllable on the interval $I$ if, for every $x_{0}, x_{1} \in \mathbb{X}$, there exists a control $u \in L^{2}(I, U)$ such that a mild solution $x$ of system (1.1) satisfies $x(b)+g(x)=x_{1}$. 


\section{Main results}

For the convenience of presentation, we list here the following hypotheses to be used later.

$\left(\mathrm{H}_{1}\right)$ The operator $A: D(A) \subset \mathbb{X} \rightarrow \mathbb{X}$ is a closed linear operator, and $-A$ generates an equicontinuous $C_{0}$-semigroup $(T(t))_{t>0}$ of uniformly bounded operators in $\mathbb{X}$, there exists a constant $M_{1}$ such that $\sup _{t \in I}\|T(t)\| \leq M_{1}$.

$\left(\mathrm{H}_{2}\right)$ The nonlinearity $f: I \times \mathbb{X} \times \mathbb{X} \times \mathbb{X} \rightarrow \mathbb{X}$ satisfies the Carathéodory type conditions, that is, $f(\cdot, x, S x, T x)$ is strong measure for all $x \in \mathbb{X}$, and $f(t, \cdot, \cdot, \cdot)$ is continuous for a.e. $t \in I$.

$\left(\mathrm{H}_{3}\right)$ For $r>0$, there exist constants $q_{1} \in[0, q)$ and functions $\varphi_{r} \in L^{1 / q_{1}}\left(I, \mathbb{R}^{+}\right)$such that for a.e. $t \in I$ and all $x \in \mathbb{X}$ satisfying $\|x\| \leq r$,

$$
\|f(t, x, S x, T x)\| \leq \varphi_{r}(t)
$$

Moreover, there exists a constant $\rho_{1}>0$ such that

$$
\liminf _{r \rightarrow+\infty} \frac{\left\|\varphi_{r}(s)\right\|_{L^{1 / q_{1}[0, b]}}}{r}=\rho_{1}<+\infty .
$$

$\left(\mathrm{H}_{4}\right)$ There exist constants $L_{i}>0(i=1,2,3)$ such that for any bounded and countable sets $D_{i} \subset E(i=1,2,3)$ and a.e. $t \in I$,

$$
\partial\left(f\left(t, D_{1}, D_{2}, D_{3}\right)\right) \leq L_{1} \partial\left(D_{1}\right)+L_{2} \partial\left(D_{2}\right)+L_{3} \partial\left(D_{3}\right) .
$$

$\left(\mathrm{H}_{5}\right)$ The nonlocal term $g: C(I, E) \rightarrow \mathbb{X}$ is compact and continuous, there exist a constant $\rho_{2}>0$ and a nondecreasing continuous function $\psi: \mathbb{R}^{+} \rightarrow \mathbb{R}^{+}$such that, for some $r>0$ and all $x \in \Omega_{r}=\left\{x \in C(I, \mathbb{X}):\|x\|_{C} \leq r\right\}$,

$$
\|g(x)\| \leq \psi(r), \quad \liminf _{r \rightarrow+\infty} \frac{\psi(r)}{r}=\rho_{2}<+\infty .
$$

$\left(\mathrm{H}_{6}\right)$ The linear operator $B: L^{2}(I, U) \rightarrow L^{1}(I, \mathbb{X})$ is bounded, $W: L^{2}(I, U) \rightarrow X$ defined by

$$
W u=\int_{0}^{b}(b-s)^{q-1} \mathcal{S}_{q}(b-s) B u(s) d s
$$

has an inverse operator $W^{-}$which takes values in $L^{2}(I, U) / \operatorname{ker} W$, and there exist two positive constants $M_{2}, M_{3}>0$ such that

$$
\|B\|_{L_{b}(U, \mathbb{X})} \leq M_{2}, \quad\left\|W^{-}\right\|_{L_{b}\left(\mathbb{X}, L^{2}(I, U) / \text { ker } W\right)} \leq M_{3} .
$$

Theorem 3.1 Assume that hypotheses $\left(\mathrm{H}_{1}\right)-\left(\mathrm{H}_{6}\right)$ are satisfied. Further assume that the following inequality holds:

$$
\begin{aligned}
& M_{1}\left\|x_{0}\right\|+M_{1} \rho_{2}+\frac{M_{1} b^{q-q_{1}}}{\Gamma(q)}\left(\frac{1-q_{1}}{q-q_{1}}\right) \rho_{1}+\frac{M_{1} M_{2}}{\Gamma(q)} \int_{0}^{t}(t-s)^{q-1} \\
& \quad \times M_{3}\left[\left\|x_{1}\right\|+M_{1}\left\|x_{0}\right\|+M_{1} \rho_{2}+\frac{M_{1} b^{q-q_{1}}}{\Gamma(q)}\left(\frac{1-q_{1}}{q-q_{1}}\right) \rho_{1}\right] d s \leq 1,
\end{aligned}
$$

then the fractional evolution system (1.1) is controllable on I. 
Proof Define the operator $Q: C(I, \mathbb{X}) \rightarrow C(I, \mathbb{X})$ as follows:

$$
\begin{aligned}
(Q x)(t)= & \mathcal{T}_{q}(t)\left(x_{0}-g(x)\right) \\
& +\int_{0}^{t}(t-s)^{q-1} \mathcal{S}_{q}(t-s)\left[f(s, x(s),(S x)(s),(T x)(s))+B u_{x}(s)\right] d s .
\end{aligned}
$$

We shall show that, using the control

$$
\begin{aligned}
u_{x}(t)= & W^{-}\left[x_{1}-\mathcal{T}_{q}(b)\left(x_{0}-g(x)\right)\right. \\
& \left.-\int_{0}^{b}(b-s)^{q-1} \mathcal{S}_{q}(b-s) f(s, x(s),(S x)(s),(T x)(s)) d s\right](t),
\end{aligned}
$$

the operator $Q$ has a fixed point, which is a mild solution of fractional evolution system (1.1). Note that $(Q x)(b)=x_{1}$ and Definition 2.4, which means that system (1.1) is controllable on $I$.

Step 1. Q maps bounded sets into bounded sets.

For any $x \in \Omega_{r}$, it follows from Lemma 2.1, hypotheses $\left(\mathrm{H}_{3}\right),\left(\mathrm{H}_{5}\right)$ and $\left(\mathrm{H}_{6}\right)$, and the Hölder inequality that

$$
\begin{aligned}
\|(Q x)(t)\| \leq & M_{1}\left\|x_{0}\right\|+M_{1}\|g(x)\|+\frac{M_{1}}{\Gamma(q)} \int_{0}^{t}(t-s)^{q-1}\|f(t, x(s),(S x)(s),(T x)(s))\| d s \\
& +\frac{M_{1}}{\Gamma(q)} \int_{0}^{t}(t-s)^{q-1}\left\|B u_{x}(s)\right\| d s \\
\leq & M_{1}\left\|x_{0}\right\|+M_{1} \psi(r)+\frac{M_{1} b^{q-q_{1}}}{\Gamma(q)}\left(\frac{1-q_{1}}{q-q_{1}}\right)\left\|\varphi_{r}\right\|_{L^{1 / q_{1}}[0, b]}+\frac{M_{1} M_{2}}{\Gamma(q)} \int_{0}^{t}(t-s)^{q-1} \\
& \times M_{3}\left[\left\|x_{1}\right\|+M_{1}\left\|x_{0}\right\|+M_{1} \psi(r)\right. \\
& \left.+\frac{M_{1} b^{q-q_{1}}}{\Gamma(q)}\left(\frac{1-q_{1}}{q-q_{1}}\right)\left\|\varphi_{r}\right\|_{L^{1 / q_{1}}[0, b]}\right] d s .
\end{aligned}
$$

By (3.1) and (3.4), we know that $\|(Q x)(t)\| \leq r$. Therefore, $Q$ maps bounded sets into bounded sets.

Step 2. $Q$ is continuous in $\Omega_{r}$.

Assume that $\left\{x_{n}\right\}_{n=1}^{\infty} \subset \Omega_{r}$ and $\lim _{n \rightarrow+\infty} x_{n}=x^{*}$. By hypotheses $\left(\mathrm{H}_{2}\right)$ and $\left(\mathrm{H}_{5}\right)$, we get

$$
\begin{aligned}
& \lim _{n \rightarrow+\infty} f\left(s, x_{n}(s),\left(S x_{n}\right)(s),\left(T x_{n}\right)(s)\right)=f(s, x(s),(S x)(s),(T x)(s)), \\
& \lim _{n \rightarrow+\infty} g\left(x_{n}\right)=g(x) .
\end{aligned}
$$

From hypothesis $\left(\mathrm{H}_{3}\right)$, we have

$$
\begin{aligned}
& (t-s)^{q-1}\left\|f\left(s, x_{n}(s),\left(S x_{n}\right)(s),\left(T x_{n}\right)(s)\right)-f(s, x(s),(S x)(s),(T x)(s))\right\| \\
& \quad \leq 2(t-s)^{q-1} \varphi_{r}(s) .
\end{aligned}
$$


Then by the Lebesgue dominated convergence theorem, we obtain that

$$
\begin{aligned}
\left\|\left(Q x_{n}\right)(t)-(Q x)(t)\right\| \leq & M_{1}\left\|g\left(x_{n}\right)-g(x)\right\| \\
& +\frac{M_{1}}{\Gamma(q)} \int_{0}^{t}(t-s)^{q-1}\left[\| f\left(s, x_{n}(s),\left(S x_{n}\right)(s),\left(T x_{n}\right)(s)\right)\right. \\
& -f(s, x(s),(S x)(s),(T x)(s)) \| \\
& \left.+\left\|B u_{x_{n}}(s)-B u_{x}(s)\right\|\right] d s \rightarrow 0, \quad n \rightarrow \infty .
\end{aligned}
$$

Therefore, $\left\|Q x_{n}-Q x\right\|_{C} \rightarrow 0$ as $n \rightarrow \infty$, that is, $Q$ is continuous.

Step 3. $Q: \Omega_{r} \rightarrow \Omega_{r}$ is equicontinuous.

For any $x \in \Omega_{r}$ and $0 \leq t_{1}<t_{2} \leq b$, we know that

$$
\begin{aligned}
(Q x)\left(t_{2}\right)-(Q x)\left(t_{1}\right)= & \left(\mathcal{T}_{q}\left(t_{2}\right)-\mathcal{T}_{q}\left(t_{1}\right)\right)\left(x_{0}-g(x)\right) \quad \text { denoted by } I_{1} \\
& +\int_{t_{1}}^{t_{2}}\left(t_{2}-s\right)^{q-1} \mathcal{S}_{q}\left(t_{2}-s\right) f(s, x(s),(S x)(s),(T x)(s)) d s
\end{aligned}
$$

denoted by $I_{2}$

$$
\begin{aligned}
& +\int_{t_{1}}^{t_{2}}\left(t_{2}-s\right)^{q-1} \mathcal{S}_{q}\left(t_{2}-s\right)+B u_{x}(s) d s \quad \text { denoted by } I_{5} \\
& +\int_{0}^{t_{1}}\left[\left(t_{2}-s\right)^{q-1}-\left(t_{1}-s\right)^{q-1}\right] f(s, x(s),(S x)(s),(T x)(s)) d s
\end{aligned}
$$

denoted by $I_{3}$

$$
\begin{aligned}
& +\int_{0}^{t_{1}}\left[\left(t_{2}-s\right)^{q-1}-\left(t_{1}-s\right)^{q-1}\right] B u_{x}(s) d s \text { denoted by } I_{6} \\
& +\int_{0}^{t_{1}}\left(t_{1}-s\right)^{q-1}\left[\mathcal{S}_{q}\left(t_{2}-s\right)-\mathcal{S}_{q}\left(t_{1}-s\right)\right] f(s, x(s),(S x)(s),(T x)(s)) d s
\end{aligned}
$$

denoted by $I_{4}$

$$
\begin{aligned}
& +\int_{0}^{t_{1}}\left(t_{1}-s\right)^{q-1}\left[\mathcal{S}_{q}\left(t_{2}-s\right)-\mathcal{S}_{q}\left(t_{1}-s\right)\right] B u_{x}(s) d s \text { denoted by } I_{7} \\
= & I_{1}+I_{2}+I_{3}+I_{4}+I_{5}+I_{6}+I_{7} .
\end{aligned}
$$

Next, we shall show that $\left\|(Q x)\left(t_{2}\right)-(Q x)\left(t_{1}\right)\right\| \rightarrow 0$ as $t_{2} \rightarrow t_{1}$. For $I_{1}$, by Lemma $2.1,\left\|I_{1}\right\| \rightarrow$ 0 as $t_{2} \rightarrow t_{1}$. For $I_{2}$, by hypothesis $\left(\mathrm{H}_{3}\right)$ and the Hölder inequality, we know that

$$
\begin{aligned}
\left\|I_{2}\right\| & \leq \frac{M_{1}}{\Gamma(q)} \int_{t_{1}}^{t_{2}}\left(t_{2}-s\right)^{q-1} \varphi_{r}(s) d s \\
& \leq \frac{M_{1}}{\Gamma(q)}\left(\int_{t_{1}}^{t_{2}}\left(t_{2}-s\right)^{(q-1) /\left(1-q_{1}\right)} d s\right)^{1-q_{1}} \times\left\|\varphi_{r}\right\|_{L^{1 / q_{1}}\left[t_{1}, t_{2}\right]} \\
& \leq \frac{M_{1}\left\|\varphi_{r}\right\|_{L^{1 / q_{1}}\left[t_{1}, t_{2}\right]}}{\Gamma(q)}\left(\frac{1-q_{1}}{q-q_{1}}\right)^{1-q_{1}}\left(t_{2}-t_{1}\right)^{q-q_{1}} .
\end{aligned}
$$


Obviously, $\left\|I_{2}\right\| \rightarrow 0$ as $t_{2} \rightarrow t_{1}$. For $I_{3}$, we have

$$
\begin{aligned}
& \left\|I_{3}\right\| \leq \frac{M_{1}}{\Gamma(q)} \int_{0}^{t_{1}}\left(\left(t_{1}-s\right)^{q-1}-\left(t_{2}-s\right)^{q-1}\right) \varphi_{r}(s) d s \\
& \leq \frac{M_{1}}{\Gamma(q)}\left(\int_{0}^{t_{1}}\left(\left(t_{1}-s\right)^{q-1}-\left(t_{2}-s\right)^{q-1}\right)^{1 /\left(1-q_{1}\right)} d s\right)^{1-q_{1}} \times\left\|\varphi_{r}\right\|_{L^{1 / q_{1}}\left[0, t_{1}\right]} \\
& \leq \frac{M_{1}}{\Gamma(q)}\left(\int_{0}^{t_{1}}\left(\left(t_{1}-s\right)^{\frac{q-1}{1-q_{1}}}-\left(t_{2}-s\right)^{\frac{q-1}{1-q_{1}}}\right) d s\right)^{1-q_{1}} \times\left\|\varphi_{r}\right\|_{L^{1 / q_{1}}[0, b]} \\
& \leq \frac{M_{1}\left\|\varphi_{r}\right\|_{L^{1 / q_{1}}[0, b]}}{\Gamma(q)}\left(\frac{1-q_{1}}{q-q_{1}}\right)^{1-q_{1}} \times\left(t_{1}^{\frac{q-q_{1}}{1-q_{1}}}-t_{2}^{\frac{q-q_{1}}{1-q_{1}}}+\left(t_{2}-t_{1}\right)^{\frac{q-q_{1}}{1-q_{1}}}\right)^{1-q_{1}} \\
& \leq \frac{2^{1-q_{1}} M_{1}\left\|\varphi_{R}\right\|_{L^{1 / q_{1}}[0, b]}}{\Gamma(q)}\left(\frac{1-q_{1}}{q-q_{1}}\right)^{1-q_{1}}\left(t_{2}-t_{1}\right)^{q-q_{1}} .
\end{aligned}
$$

So we get that $\left\|I_{3}\right\| \rightarrow 0$ as $t_{2} \rightarrow t_{1}$. For $t_{1}=0,0<t_{2} \leq b$, we have $\left\|I_{4}\right\|=0$. For $t_{1}>0$ and $\varepsilon>0$ small enough, by hypothesis $\left(\mathrm{H}_{3}\right)$, the Lebesgue dominated convergence theorem, and the equicontinuity of $T(t)$, we get that

$$
\begin{aligned}
\left\|I_{4}\right\| \leq & \int_{0}^{t_{1}-\varepsilon}\left(t_{1}-s\right)^{q-1}\left[\mathcal{S}_{q}\left(t_{2}-s\right)-\mathcal{S}_{q}\left(t_{1}-s\right)\right] f(s, x(s),(S x)(s),(T x)(s)) d s \\
& +\int_{t_{1}-\varepsilon}^{t_{1}}\left(t_{1}-s\right)^{q-1}\left[\mathcal{S}_{q}\left(t_{2}-s\right)-\mathcal{S}_{q}\left(t_{1}-s\right)\right] f(s, x(s),(S x)(s),(T x)(s)) d s \\
\leq & \int_{0}^{t_{1}-\varepsilon}\left(t_{1}-s\right)^{q-1}\left[\mathcal{S}_{q}\left(t_{2}-s\right)-\mathcal{S}_{q}\left(t_{1}-s\right)\right] f(s, x(s),(S x)(s),(T x)(s)) d s \\
& +\frac{2 M_{1}}{\Gamma(q)} \int_{t_{1}-\varepsilon}^{t_{1}}\left(t_{1}-s\right)^{q-1} \varphi_{r}(s) d s \\
\leq & \int_{0}^{t_{1}-\varepsilon}\left(t_{1}-s\right)^{q-1}\left[\mathcal{S}_{q}\left(t_{2}-s\right)-\mathcal{S}_{q}\left(t_{1}-s\right)\right] f(s, x(s),(S x)(s),(T x)(s)) d s \\
& +\frac{2 M_{1}\left\|\varphi_{R}\right\|_{L^{1 / q}[0, b]}}{\Gamma(q)}\left(\frac{1-q_{1}}{q-q_{1}}\right)^{1-q_{1}} \varepsilon^{q-q_{1}} .
\end{aligned}
$$

Then $\left\|I_{4}\right\| \rightarrow 0$ as $t_{2} \rightarrow t_{1}, \varepsilon \rightarrow 0$. In a similar way, for $I_{5}, I_{6}, I_{7}, I_{8}$, we obtain

$$
\begin{aligned}
\left\|I_{5}\right\| \leq & \frac{M_{1} M_{2}}{\Gamma(q)}\left(\frac{1-q_{1}}{q-q_{1}}\right)^{1-q_{1}}\left(t_{2}-t_{1}\right)^{q-q_{1}}\left(\int_{t_{1}}^{t_{2}}\left\|u_{x}\right\|^{1 / q_{1}} d s\right)^{q_{1}}, \\
\left\|I_{6}\right\| \leq & \frac{2^{1-q_{1}} M_{1} M_{2}}{\Gamma(q)}\left(\frac{1-q_{1}}{q-q_{1}}\right)^{1-q_{1}}\left(t_{2}-t_{1}\right)^{q-q_{1}}\left(\int_{0}^{t_{1}}\left\|u_{x}\right\|^{1 / q_{1}} d s\right)^{q_{1}}, \\
\left\|I_{7}\right\| \leq & M_{2}\left(\int_{0}^{t_{1}-\varepsilon}\left(t_{1}-s\right)^{q-1}\left[\mathcal{S}_{q}\left(t_{2}-s\right)-\mathcal{S}_{q}\left(t_{1}-s\right)\right] f(s, x(s),(S x)(s),(T x)(s)) d s\right. \\
& \left.+\frac{2 M_{1}}{\Gamma(q)}\left(\frac{1-q_{1}}{q-q_{1}}\right)^{1-q_{1}} \varepsilon^{q-q_{1}}\right)^{1-q_{1}}\left(\int_{0}^{t_{1}}\left\|u_{x}\right\|^{1 / q_{1}} d s\right)^{q_{1}} .
\end{aligned}
$$

It can be easily seen that $I_{5}, I_{6}$ and $I_{7}$ tend to 0 . Therefore, for any $x \in \Omega_{r}, \|(Q x)\left(t_{2}\right)-$ $(Q x)\left(t_{1}\right) \| \rightarrow 0$ as $t_{2} \rightarrow t_{1}$, which means that $Q: \Omega_{r} \rightarrow \Omega_{r}$ is equicontinuous.

Step 4. $Q: F \rightarrow F$ is a convex-power condensing operator. 
Let $F=\overline{c o} Q\left(\Omega_{r}\right)$, where $\overline{c o}$ means the closure of convex hull. It is easy to see that $Q$ maps $F$ into itself and $F \subset C(J, E)$ is equicontinuous. Next, we shall show that there exists a positive integer $n_{0}$ such that for any bounded and nonprecompact subset $D \subset F, x_{0} \in F$,

$$
\partial\left(Q^{\left(n_{0}, x_{0}\right)}(D)\right)<\partial(D)
$$

Obviously, $Q^{\left(n, x_{0}\right)}(D) \subset \Omega_{r}$ is also equicontinuous for $D \subset F$ and $x_{0} \subset F$. Therefore, from Lemma 2.4, we attain

$$
\partial\left(Q^{\left(n, x_{0}\right)}(D)\right)=\max _{t \in I} \partial\left(Q^{\left(n, x_{0}\right)}(D)(t)\right), \quad n=1,2, \ldots
$$

Note that by Lemma 2.6, there exists a countable set $D_{1}=\left\{x_{n}^{1}\right\} \subset D$ such that

$$
\partial(Q(D)(t)) \leq 2 \partial\left(Q\left(D_{1}\right)(t)\right)
$$

By $\left(\mathrm{H}_{4}\right)$ and $\left(\mathrm{H}_{6}\right)$, we have

$$
\begin{aligned}
\partial\left(u_{x_{n}^{1}}(t)\right)= & \partial\left\{W ^ { - } \left[x_{1}-\mathcal{T}_{q}(b)\left(x_{0}-g\left(x_{n}^{1}\right)\right)\right.\right. \\
& \left.\left.-\int_{0}^{b}(b-s)^{q-1} \mathcal{S}_{q}(b-s) f\left(s, x_{n}^{1}(s),\left(S x_{n}^{1}\right)(s),\left(T x_{n}^{1}\right)(s)\right)\right](t)\right\} \\
\leq & M_{3} \frac{4 M_{1}}{\Gamma(q)} \int_{0}^{b}(b-s)^{q-1}\left[L_{1} \partial\left(D_{1}(s)\right)+L_{2} \partial\left(\left(S D_{1}\right)(s)\right)+L_{3} \partial\left(\left(T D_{1}\right)(s)\right)\right] d s \\
\leq & M_{3} \frac{4 M_{1}\left(L_{1}+b K_{0} L_{2}+b H_{0} L_{3}\right) b^{q}}{\Gamma(1+q)} \partial\left(D_{1}\right) .
\end{aligned}
$$

Suppose instead

$$
\begin{aligned}
& L=M_{3} \frac{4 M_{1}\left(L_{1}+b K_{0} L_{2}+b H_{0} L_{3}\right) b^{q}}{\Gamma(1+q)}, \\
& K_{0}=\max _{(t, s) \in \Delta_{1}}|k(t, s)|, \quad H_{0}=\max _{(t, s) \in \Delta_{2}}|h(t, s)|,
\end{aligned}
$$

then

$$
\begin{aligned}
\partial\left(\left(Q^{\left(1, x_{0}\right)} D\right)(t)\right)= & \partial((Q D)(t)) \leq 2 \partial\left(\left(Q D_{1}\right)(t)\right) \\
\leq & 2 \partial\left(\mathcal{T}_{q}(t) g\left(x_{n}^{1}\right)\right. \\
& \left.+\int_{0}^{t}(t-s)^{q-1} \mathcal{S}_{q}(t-s)\left[f\left(s, x_{n}^{1}(s),\left(S x_{n}^{1}\right)(s),\left(T x_{n}^{1}\right)(s)\right)+B u_{x_{n}^{1}}(s)\right] d s\right) \\
\leq & \frac{4 M_{1}}{\Gamma(q)} \int_{0}^{t}(t-s)^{q-1} \partial\left(f\left(s, x_{n}^{1}(s),\left(S x_{n}^{1}\right)(s),\left(T x_{n}^{1}\right)(s)\right)+B u_{x_{n}^{1}}(s)\right) d s \\
\leq & \frac{4 M_{1}}{\Gamma(q)} \int_{0}^{t}(t-s)^{q-1}\left(L_{1}+b K_{0} L_{2}+b H_{0} L_{3}+L\right) \partial\left(D_{1}(s)\right) d s \\
\leq & \frac{4 M_{1}\left(L_{1}+b K_{0} L_{2}+b H_{0} L_{3}+L\right) t^{q}}{\Gamma(1+q)} \partial(D) .
\end{aligned}
$$


There exists a countable set $D_{2}=\left\{x_{n}^{2}\right\} \subset \overline{c o}\left\{\left(Q^{\left(1, x_{0}\right)} D\right), x_{0}\right\}$ such that

$$
\begin{aligned}
& \partial\left(Q\left(\overline{c o}\left\{\left(Q^{\left(1, x_{0}\right)} D\right), x_{0}\right\}\right)(t)\right) \leq 2 \partial\left(\left(Q D_{2}\right)(t)\right), \\
& \partial\left(\left(Q^{\left(2, x_{0}\right)} D\right)(t)\right) \\
& =\partial\left(Q\left(\overline{c o}\left\{\left(Q^{\left(1, x_{0}\right)} D\right), x_{0}\right\}\right)(t)\right) \leq 2 \partial\left(\left(Q D_{2}\right)(t)\right) \\
& \leq 2 \partial\left(\mathcal{T}_{q}(t) g\left(x_{n}^{2}\right)\right. \\
& \left.+\int_{0}^{t}(t-s)^{q-1} \mathcal{S}_{q}(t-s)\left[f\left(s, x_{n}^{2}(s),\left(S x_{n}^{2}\right)(s),\left(T x_{n}^{2}\right)(s)\right)+B u_{x_{n}^{2}}(s)\right] d s\right) \\
& \leq \frac{4 M_{1}}{\Gamma(q)} \int_{0}^{t}(t-s)^{q-1} \partial\left(f\left(s, x_{n}^{2}(s),\left(S x_{n}^{2}\right)(s),\left(T x_{n}^{2}\right)(s)\right)+B u_{x_{n}^{2}}(s)\right) d s \\
& \leq \frac{4 M_{1}}{\Gamma(q)} \int_{0}^{t}(t-s)^{q-1}\left[L_{1} \partial\left(D_{2}(s)\right)+L_{2} \partial\left(\left(S D_{2}\right)(s)\right)\right. \\
& \left.+L_{3} \partial\left(\left(T D_{2}\right)(s)\right)+L \partial\left(D_{2}\right)\right] d s \\
& \leq \frac{4 M_{1}}{\Gamma(q)} \int_{0}^{t}(t-s)^{q-1}\left(L_{1}+b K_{0} L_{2}+b H_{0} L_{3}+L\right) \partial\left(D_{2}(s)\right) d s \\
& \leq \frac{4 M_{1}}{\Gamma(q)} \int_{0}^{t}(t-s)^{q-1}\left(L_{1}+b K_{0} L_{2}+b H_{0} L_{3}+L\right) \partial\left(\overline{c o}\left\{\left(Q^{\left(1, x_{0}\right)} D\right), x_{0}\right\}(s)\right) d s \\
& \leq \frac{\left[4 M_{1}\left(L_{1}+b K_{0} L_{2}+b H_{0} L_{3}+L\right) t^{q}\right]^{2}}{\Gamma(q) \Gamma(1+q)} \int_{0}^{t}(t-s)^{q-1} s^{q} \partial(D) d s \\
& =\frac{\left[4 M_{1}\left(L_{1}+b K_{0} L_{2}+b H_{0} L_{3}+L\right) t^{q}\right]^{2}}{\Gamma(1+2 q) \mathbf{B}(1+q, q)} \int_{0}^{1}(1-s)^{q-1} s^{q} d s \partial(D) \\
& =\frac{\left[4 M_{1}\left(L_{1}+b K_{0} L_{2}+b H_{0} L_{3}+L\right) t^{q}\right]^{2}}{\Gamma(1+2 q)} \partial(D),
\end{aligned}
$$

where $\mathbf{B}(p, q)=\int_{0}^{1} s^{p-1}(1-s)^{q-1} d s$ is the beta function. Suppose instead

$$
\partial\left(\left(Q^{\left(k, x_{0}\right)} D\right)(t)\right) \leq \frac{\left[4 M_{1}\left(L_{1}+b K_{0} L_{2}+b H_{0} L_{3}+L\right) t^{q}\right]^{k}}{\Gamma(1+k q)} \partial(D), \quad \forall t \in I .
$$

Similarly, we get that

$$
\begin{aligned}
& \partial\left(\left(Q^{\left(k+1, x_{0}\right)} D\right)(t)\right) \\
&= \partial\left(Q\left(\overline{c o}\left\{\left(Q^{\left(k, x_{0}\right)} D\right), x_{0}\right\}\right)(t)\right) \leq 2 \partial\left(\left(Q D_{k+1}\right)(t)\right) \\
& \leq \frac{4 M_{1}}{\Gamma(q)} \int_{0}^{t}(t-s)^{q-1} \partial\left(f\left(s, x_{n}^{k+1}(s),\left(S x_{n}^{k+1}\right)(s),\left(T x_{n}^{k+1}\right)(s)\right)+B u_{x_{n}^{k+1}}(s)\right) d s \\
& \leq \frac{4 M_{1}}{\Gamma(q)} \int_{0}^{t}(t-s)^{q-1}\left[L_{1} \partial\left(D_{k+1}(s)\right)+L_{2} \partial\left(\left(S D_{k+1}\right)(s)\right)\right. \\
&\left.+L_{3} \partial\left(\left(T D_{k+1}\right)(s)\right)+L \partial\left(D_{k+1}(s)\right)\right] d s \\
& \leq \frac{4 M_{1}}{\Gamma(q)} \int_{0}^{t}(t-s)^{q-1}\left(L_{1}+b K_{0} L_{2}+b H_{0} L_{3}+L\right) \partial\left(D_{k+1}(s)\right) d s
\end{aligned}
$$




$$
\begin{aligned}
& \leq \frac{4 M_{1}}{\Gamma(q)} \int_{0}^{t}(t-s)^{q-1}\left(L_{1}+b K_{0} L_{2}+b H_{0} L_{3}+L\right) \partial\left(\overline{c o}\left\{\left(Q^{\left(k, x_{0}\right)} D\right), x_{0}\right\}(s)\right) d s \\
& \leq \frac{\left[4 M_{1}\left(L_{1}+b K_{0} L_{2}+b H_{0} L_{3}+L\right) t^{q}\right]^{k+1}}{\Gamma(q) \Gamma(1+k q)} \int_{0}^{t}(t-s)^{q-1} s^{k q} \partial(D) d s \\
& =\frac{\left[4 M_{1}\left(L_{1}+b K_{0} L_{2}+b H_{0} L_{3}+L\right) t^{q}\right]^{k+1}}{\Gamma(1+(k+1) q) \mathbf{B}(1+q, q)} \int_{0}^{1}(1-s)^{q-1} s^{k q} d s \partial(D) \\
& =\frac{\left[4 M_{1}\left(L_{1}+b K_{0} L_{2}+b H_{0} L_{3}+L\right) t^{q}\right]^{k+1}}{\Gamma(1+(k+1) q)} \partial(D) .
\end{aligned}
$$

Then by the principle of mathematical induction, for any $n>0$, we attain

$$
\partial\left(\left(Q^{\left(n, x_{0}\right)} D\right)(t)\right)=\frac{\left[4 M_{1}\left(L_{1}+b K_{0} L_{2}+b H_{0} L_{3}+L\right) t^{q}\right]^{n}}{\Gamma(1+n q)} \partial(D) .
$$

So we have

$$
\begin{aligned}
\partial\left(\left(Q^{\left(n, x_{0}\right)} D\right)\right) & =\max _{t \in I} \partial\left(\left(Q^{\left(n, x_{0}\right)} D\right)(t)\right) \\
& \leq \frac{\left[4 M_{1}\left(L_{1}+b K_{0} L_{2}+b H_{0} L_{3}+L\right) b^{q}\right]^{n}}{\Gamma(1+n q)} \partial(D) .
\end{aligned}
$$

From [14], there exists a large enough positive integer $n_{0}$ such that

$$
\frac{\left[4 M_{1}\left(L_{1}+b K_{0} L_{2}+b H_{0} L_{3}+L\right) b^{q}\right]^{n_{0}}}{\Gamma(1+n q)}<1 .
$$

Hence

$$
\partial\left(\left(Q^{\left(n, x_{0}\right)} D\right)\right)<\partial(D)
$$

Therefore $Q: F \rightarrow F$ is a convex-power condensing operator, $Q$ has at least one fixed point, which is a mild solution of system (1.1). By Definition 2.4, system (1.1) is controllable on $I$.

In order to obtain more controllability results, we replace conditions $\left(\mathrm{H}_{3}\right)$ and $\left(\mathrm{H}_{5}\right)$ by the following hypotheses:

$\left(\mathrm{H}_{3}^{\prime}\right)$ There exist a function $\varphi \in L^{1 / q_{1}}\left(I, \mathbb{R}^{+}\right), q_{1} \in[0, q)$ and a nondecreasing continuous function $\Psi: \mathbb{R}^{+} \rightarrow \mathbb{R}^{+}$such that

$$
\|f(t, x, S x, T x)\| \leq \varphi(t) \Psi(\|x\|)
$$

for all $x \in \mathbb{X}$ and a.e. $t \in I$.

$\left(\mathrm{H}_{5}^{\prime}\right)$ The nonlocal term $g: C(I, E) \rightarrow \mathbb{X}$ is compact and continuous, and there exist constants $d, c>0$ such that

$$
\|g(u)\| \leq d\|x\|_{C}+c .
$$


Theorem 3.2 Assume that hypotheses $\left(\mathrm{H}_{1}\right)-\left(\mathrm{H}_{2}\right),\left(\mathrm{H}_{3}^{\prime}\right),\left(\mathrm{H}_{4}\right)$ and $\left(\mathrm{H}_{5}^{\prime}\right)$ are satisfied. Further assume that there exists a constant $R$ and the following inequality holds:

$$
\begin{aligned}
& {\left[M_{1}\left\|x_{0}\right\|+M_{1} d R+M_{1} c+\frac{M_{1} \Psi(R) b^{1-q_{1}}}{\Gamma(q)}\left(\frac{1-q_{1}}{q-q_{1}}\right)^{1-q_{1}}\|\varphi\|_{L^{1 / q_{1}}[0, b]}\right]+\frac{M_{1} M_{2} M_{3}}{\Gamma(q)}} \\
& \quad \times\left[\left\|x_{1}\right\|+M_{1}\left\|x_{0}\right\|+M_{1} d R+M_{1} c+\frac{M_{1} \Psi(R) b^{1-q_{1}}}{\Gamma(q)}\left(\frac{1-q_{1}}{q-q_{1}}\right)^{1-q_{1}}\|\varphi\|_{L^{1 / q_{1}}[0, b]}\right] \\
& \quad \times b^{1-q_{1}}\left(\frac{1-q_{1}}{q-q_{1}}\right)^{1-q_{1}} \\
& \leq R
\end{aligned}
$$

then the fractional evolution system (1.1) is controllable on I.

Proof By the definition of $u_{x}$ and the hypotheses we imposed, we have

$$
\begin{aligned}
& \frac{M_{1}}{\Gamma(q)} \int_{0}^{t}(t-s)^{q-1}\left\|B u_{x}(s)\right\| d s \\
& \leq \frac{M_{1} M_{2} M_{3}}{\Gamma(q)} \int_{0}^{t}(t-s)^{q-1}\left[\left\|x_{1}\right\|+M_{1}\left\|x_{0}\right\|+M_{1} d\|x\|_{C}+M_{1} c\right. \\
& \left.\quad+\frac{M_{1} \Psi(R)}{\Gamma(q)} \int_{0}^{b}(b-s)^{q-1} \varphi(\tau) d \tau\right](s) d s \\
& \leq \frac{M_{1} M_{2} M_{3}}{\Gamma(q)}\left[\left\|x_{1}\right\|+M_{1}\left\|x_{0}\right\|+M_{1} d R+M_{1} c\right. \\
& \left.\quad+\frac{M_{1} \Psi(R) b^{1-q_{1}}}{\Gamma(q)}\left(\frac{1-q_{1}}{q-q_{1}}\right)^{1-q_{1}}\|\varphi\|_{L^{1 / q_{1}}[0, b]}\right] \\
& \quad \times b^{1-q_{1}}\left(\frac{1-q_{1}}{q-q_{1}}\right)^{1-q_{1}} \cdot
\end{aligned}
$$

From (3.26) we have

$$
\begin{aligned}
\|(Q x)(t)\| \leq & M_{1}\left\|x_{0}\right\|+M_{1}\|g(x)\|+\frac{M_{1}}{\Gamma(q)} \int_{0}^{t}(t-s)^{q-1}\|f(s, x(s),(S x)(s),(T x)(s))\| d s \\
& +\frac{M_{1}}{\Gamma(q)} \int_{0}^{t}(t-s)^{q-1}\left\|B u_{x}(s)\right\| d s \\
\leq & {\left[M_{1}\left\|x_{0}\right\|+M_{1} d R+M_{1} c\right.} \\
& \left.\quad+\frac{M_{1} \Psi(R) b^{1-q_{1}}}{\Gamma(q)}\left(\frac{1-q_{1}}{q-q_{1}}\right)^{1-q_{1}}\|\varphi\|_{L^{1 / q_{1}}[0, b]}\right]+\frac{M_{1} M_{2} M_{3}}{\Gamma(q)} \\
& \times\left[\left\|x_{1}\right\|+M_{1}\left\|x_{0}\right\|+M_{1} d R+M_{1} c\right. \\
& \left.\quad+\frac{M_{1} \Psi(R) b^{1-q_{1}}}{\Gamma(q)}\left(\frac{1-q_{1}}{q-q_{1}}\right)^{1-q_{1}}\|\varphi\|_{L^{1 / q_{1}}[0, b]}\right] \times b^{1-q_{1}}\left(\frac{1-q_{1}}{q-q_{1}}\right)^{1-q_{1}} \\
\leq & R . \quad
\end{aligned}
$$


Using a similar method as in the previous proof, we can get that the fractional evolution system (1.1) is controllable on $I$.

\section{An example}

Example 4.1 Consider the following fractional control system governed by a fractional partial differential equation:

$$
\left\{\begin{array}{l}
\frac{\partial^{q} z(t, y)}{\partial t^{q}}=\frac{\partial^{2} z(t, y)}{\partial y^{2}}+F(t, z(t, y), S z(s, y), T z(s, y))+\mu(t, y) \\
\quad t \in[0,1], y \in(-\infty,+\infty) \\
z(0, y)=z_{0}(y)+\frac{1}{t^{1 / 2}} \sin z(t, y) \\
z(t,-\infty)=z(t,+\infty)=0
\end{array}\right.
$$

where $\frac{\partial^{q}}{\partial t^{q}}$ is a Caputo fractional partial derivative of order $0<q<1, F$ is a given function.

$$
S z(t, y)=\int_{0}^{t} k(t, s) z(s, y) d s, \quad T z(t, y)=\int_{0}^{b} h(t, s) z(s, y) d s
$$

$\mu:[0,1] \times(-\infty,+\infty) \rightarrow(-\infty,+\infty)$ is continuous. Let $\mathbb{X}=U=L^{2}(-\infty,+\infty)$, assume that the operator $A$ is defined by $A w=w^{\prime \prime}$, with the domain $D(A)=\left\{w(\cdot) \in L^{2}(-\infty,+\infty), w^{\prime}, w^{\prime \prime} \in\right.$ $\left.L^{2}(-\infty,+\infty)\right\}$. It is well known that $A$ is the infinitesimal generator of a differentiable (equicontinuity) semigroup $T(t)(t>0)$ but not compact in $\mathbb{X}$, which is given by

$$
(T(t) x)(y)= \begin{cases}\int_{-\infty}^{+\infty} G(t, y-s) x(s) d s, & t>0, \\ x(y), & t=0\end{cases}
$$

where $G(t, y)=\frac{1}{\sqrt{4 \pi t}} e^{-\frac{y^{2}}{4 t}}(t>0,-\infty<y<+\infty)$. Moreover, it is easy to get that

$$
\sup _{t \in[0,+\infty)}\|T(t)\|_{L_{b}(\mathbb{X})} \leq 1
$$

Put $x(t)(y)=z(t, y), u(t)(y)=\mu(t, y)$, define the operator $B: U \rightarrow \mathbb{X}$ by $B u(t)(y)=\mu(t, y)$, $-\infty<y<+\infty$. Then, with the appropriate choice of $A, B, f$ and $g$, the fractional evolution system (4.1) can be written in the form of (1.1). The following conditions hold:

$$
W u:=\int_{0}^{b}(b-s)^{q-1} \mathcal{S}_{q}(b-s) B u(s) d s
$$

has a bounded invertible operator $W^{-}$defined by $L^{2}([0, b] ; U) /$ ker $W$. If we can verify that all the conditions of Theorem 3.1 and inequality (3.1) are satisfied, the fractional control system (1.1) is controllable on $[0,1]$, then the fractional control system (4.1) is controllable.

\section{Conclusions}

In this paper, using the uniform boundedness, equicontinuity of an operator semigroup and a fixed theorem with respect to a convex-power condensing operator, we have obtained the controllability of abstract fractional evolution control systems in a Banach space. It is well known that the compactness conditions of the operator semigroup can be weakened to equicontinuity. However, we have not only implemented a concrete assumption on the compactness condition via a parameter estimator but also removed the 
estimated parameter constraints; the sufficient conditions of controllability for various semilinear evolution systems are hence weakened. The conclusion of this paper is one of the most important developments in the aspect of imposing the necessary condition of controllability.

\section{Competing interests}

The authors declare that they have no competing interests.

\section{Authors' contributions}

The authors have contributed to this work on an equal basis. All authors read and approved the final manuscript.

\section{Acknowledgements}

The authors thank the anonymous referees and handing editor of the manuscript for their valuable suggestions and fruitful comments.

\section{Received: 24 February 2014 Accepted: 10 July 2014 Published: 18 Aug 2014}

\section{References}

1. Yan, Z: Controllability of fractional order partial neutral functional integrodifferential inclusions with infinite delay. J. Franklin Inst. 348, 2156-2173 (2011)

2. Balachandran, K, Park, JY: Controllability of fractional integrodifferential systems in Banach spaces. Nonlinear Anal. Hybrid Syst. 3, 363-367 (2009)

3. Chang, YK, Chalishajar, DN: Controllability of mixed Volterra-Fredholm type integro-differential inclusions in Banach spaces. J. Franklin Inst. 345, 499-507 (2008)

4. Chalishajar, DN: Controllability of mixed Volterra-Fredholm type integrodifferential systems in Banach space. J. Franklin Inst. 344, 12-21 (2007)

5. Hernández, EM, O’Regan, D: Controllability of Volterra-Fredholm type systems in Banach spaces. J. Franklin Inst. 346 95-101 (2009)

6. Wang, F, Liu, Z, Li, J: Complete controllability of fractional neutral differential systems in abstract space. Abstr. Appl. Anal. 2013, Article ID 529025 (2013)

7. Fečkan, M, Wang, J, Zhou, Y: Controllability of fractional functional evolution equations of Sobolev type via characteristic solution operators. J. Optim. Theory Appl. 156, 79-95 (2013)

8. Ji, S, Li, G, Wang, M: Controllability of impulsive differential systems with nonlocal conditions. Appl. Math. Comput. 217, 6981-6989 (2011)

9. Machado, JA, Ravichandran, C, Rivero, M, Trujillo, JJ: Controllability results for impulsive mixed type functional integrodifferential evolution equations with nonlocal conditions. Fixed Point Theory Appl. 2013, Article ID 66 (2013)

10. Zhu, L, Huang, Q, Li, G: Abstract semilinear evolution equations with convex power condensing operators. J. Funct. Spaces Appl. 2013, Article ID 473876 (2013)

11. Xue, X: Nonlocal nonlinear differential equations with a measure of noncompactness in Banach spaces. Nonlinear Anal. 70, 2593-2601 (2009)

12. Ahmad, B, Malar, K, Karthikeyan, K: A study of nonlocal problems of impulsive integrodifferential equations with measure of noncompactness. Adv. Differ. Equ. 2013, Article ID 205 (2013)

13. Wang, J, Zhou, Y: Complete controllability of fractional evolution systems. Commun. Nonlinear Sci. Numer. Simul. 17, 4346-4355 (2012)

14. Chen, $\mathrm{P}, \mathrm{Li}, \mathrm{Y}$ : Nonlocal problem for fractional evolution equations of mixed type with the measure of noncompactness. Abstr. Appl. Anal. 2013, Article ID 784816 (2013)

15. Podlubny, I: Fractional Differential Equations. Academic Press, San Diego (1999)

16. Miller, KS, Ross, B: An Introduction to Fractional Calculus and Differential Equations. Wiley, New York (1993)

17. Deimling, K: Nonlinear Functional Analysis. Springer, New York (1985)

18. Banaś, J, Goebel, K: Measures of Noncompactness in Banach Spaces. Lecture Notes in Pure and Applied Mathematics, vol. 60. Dekker, New York (1980)

19. Heinz, HP: On the behaviour of measure of noncompactness with respect to differentiation and integration of vector valued functions. Nonlinear Anal., Theory Methods Appl. 7(12), 1351-1371 (1983)

20. Sun, J, Zhang, X: The fixed point theorem of convex-power condensing evolution equations. Acta Math. Sin. 48(3), 439-446 (2005) (in Chinese)

10.1186/1029-242X-2014-292

Cite this article as: Qin et al.: Controllability problem for fractional integrodifferential evolution systems of mixed type with the measure of noncompactness. Journal of Inequalities and Applications 2014, 2014:292 